\title{
Editorial
}

\section{Annals of Neurosclences}

\section{IAN 2017 conference in Odisha}

\author{
Sushant Kaushal ${ }^{\mathrm{a}} \quad$ Gurkeerat Kaur $^{\mathrm{a}} \quad$ Abdul Ghani $^{\mathrm{b}} \quad$ Navneet Kaur ${ }^{\mathrm{d}}$ \\ Paul Balic Akshay Anand ${ }^{a}$ \\ ${ }^{a}$ Department of Neurology, Neuroscience Research Lab, Postgraduate Institute of Medical Education and \\ Research, Chandigarh, India; ${ }^{b}$ Department of histopathology, Postgraduate Institute of Medical Education \\ and Research, Chandigarh, India; ${ }^{C}$ Department of Biophysics, Panjab University, Chandigarh, India; ${ }^{\mathrm{d}}$ Department \\ of Physical Education, Panjab University, Chandigarh, India
}

The XXXV Annual Meeting of Indian Academy of Neurosciences 2017 was held from October 29 to 31, 2017 in Cuttack, Odisha. The theme of the meeting was "Translational Neurosciences and its Application in Protection of Mental Health". The students from Neuroscience Research Lab, PGIMER Chandigarh participated in this workshop and gathered knowledge from the talks of different neuroscientists.

On the first day, that is, October 29, all IAN members gathered near the auditorium, and attended the welcome address by Dr. Lipika Patnaik and Dr. Tisa from Kyoto University. Dr. N.R. Jana from NBRC, Manesar presented her research paper in which she explained the rescue of altered protein homeostasis in a mouse model of Huntington's disease. In the evening address, the lecture of Dr. S.K. Trigun was held on the subject "sirtuins and epigenetic pathogenesis of excitotoxic brain disorders" in which he concluded that the activation of Sirt-1 and Sirt3 could be a relevant strategy to ameliorate TNF- $\alpha$ led excitotoxic neurodegenerative condition in the rat brain. The results demonstrate that the declined level of Sirt-1 in the cerebral cortex and cerebellum associates with the neuroinflammation o(TNF- $\alpha$ and NFkB) led HE patho- genesis reflected by the transition of neuroprotective NR1/NR2A pro-inflammatory factors, neurodegenerative combinations of NMDAR composition towards a neurodegenerative NR1/NR2B combination. Dr. A. Chattopadhay, from CCMB, Hyderabad, described the interaction of Membrane Cholesterol with G protein Coupled receptor in which the dependency of serotoni$\mathrm{n} 1 \mathrm{~A}$ receptor with the $\mathrm{pH}$, ionic strength and temperature was found to enhance the interaction of brain cholesterol. In that context, they reported the presence of cholesterol recognition/interaction amino acid consensus motifs in the seotnin $1 \mathrm{~A}$ receptor. They also showed the utilizing homo-FRET that the serotonin $1 \mathrm{~A}$ receptoris constitutively oligomerized in live cells, with the possibility of higher order oligomers of the receptor. The talk by Dr. Deepak Kumar Sarkar that "alcohol induced epigenetic modifications of stress regulatory genes transmit via germilne" was discussed. He showed that the effect of alcohol in the epigenetic modulation of POMC gene led to the transgenerational increase in the level of stress hormones. They have created 2 different mice models to analyse this study; in a result they found that maternal alcohol feeding significantly affects the POMC gene

\section{KARGER}

(c) 2018 S. Karger AG, Basel

E-Mail karger@karger.com

www.karger.com/aon
Akshay Anand, $\mathrm{PhD}$

Neuroscience Research Lab, Department of Neurology

Postgraduate Institute of Medical Education and Research

Chandigarh 160012 (India)

E-Mail akshaylanand@ rediffmail.com 
methylation, gene expression and POMC endophenotypes. These findings show that alcohol-induced changes in some of the epigenetic machinery are transmitted across generations via germline.

Dr. K.P. Mohana Kumar from IUCBR, Kottayam, talked about Mitochondrial Neuronal Cell Lines: Creation, Characterization and Validation as Cellular Models of Neurological Diseases. Cell lines were created as a model to mimic neurological diseases like Parkinson disease from human cells by cybrid formation and they have checked the absence or presence of mitochondrial energy crisis in neurodegenerative disorders. They have created cells from the human neuroblastoma cell lines, which show changes in the expression of several mitochondrial genes, decline in mitochondrial respiratory activity, decreased membrane potential and altered morphology. Apart from that, they have created the autism spectrum disorders and attention deficit hyperactivity disorders cybrids and demonstrated the absence or presence of mitochondrial energy crisis in these orders to make these suitable for drug screening against the diseases and to establish the usefulness of this cellular model for studying mitochondrial dysfunctions of various neurological disorders. Dr. S.C. Bose Chinnathambi from NCL, Pune, spoke on "Microglial Internalization and Post-Translational Modifications of Tau" explaining tau as a pathological feature in $\mathrm{AD}$, its propagation and internalization by microglia, he showed cell co-culture by adding tau in monomeric and aggregated form to study internalization by microglia and its toxicity. The study concluded that small length aggregates are more toxic than long length tau.

Dr. Pankaj Seth demonstrated that the cellular and molecular mechanism mediated by astrocytes involved HIV-1 Neuropathogenesis. They explored the cellular and molecular mechanisms of astrocyte-mediated neuronal damage by studying the role of ATP-mediated neuronal damage in primary cultures of human brain cells and validated the in vitro findings in autopsy brain sections of AIDS patients. Their cell culture study showed that VDAC-1 in astrocytes releases ATP as gliotransmitter and mediate neuronal damage. The talk by RV Omkumar from RGCB, Thiruvanathapuram, explained the role of CaM Kinsae II in learning and memory using in-vitro study to understand its biochemical and structural regulation by its ligand GluN2B, which causes the dephosphorlyation of CaMKII. They have used CaM kinaseII in such an in vitro system to understand its biochemical and structural regulation by its ligands, the NMDA receptor subunit, GluN2B. They found that CaMKII is allosteri- cally modulated by GluN2B. Mutants of CaMKII, E96A and $\mathrm{H} 282 \mathrm{~A}$ were used as tools to test the role of allosteric modulations of CaMKII under physiological conditions.

Sunita Mittal from AIIMS, Rishikesh, presented her research work on memory and its opponent Alzheimer's disease, in which she described memory, its types, processing, amnesia and diagnosis of AD in CSF, marker using imaging techniques. In another lecture, Dr. A.B. Pant, IITR, Lucknow, showed the efficacy of induced pluripotent stem cells (IPSCs) in the developmental neurotoxicity. His talk on "In vitro models of developmental neurotoxicity: A journey with human cord blood stem cells" represented 2-D and 3D cultures of IPSCs. He characterized various cells in-vitro and in-vivo for iPSCs and established 3D organoid structures to be used as a model for drug testing. Parul Bali, from Panjab University, had her poster session at late afternoon on "Optimization of Amyloid $\beta$ dose for establishing memory deficit mouse model of Alzheimer's disease". A workshop was held by a neuro-artist Mr. Vinod Mehta in order to sensitize the scientific community towards science - arts convergence and its role in managing neuronal disorders.

On October 30, we attended the lecture of Dr. Serge Picaud, Paris, on visual restoration by retinal prostheses and optogenetic therapy in non-human primate models. He discussed new photovoltaic retinal implants and optogenetic therapy on non-human primates to improve further current strategies for restoring vision. Dr. S.K. Hota, DIHAR, Leh-Ladakh, presented his talk on improving neurovascular coupling to ameliorate hypobaric hypoxia-induced cognitive impairment. Then, the talk started on "Perturbed stress granules dynamics in motor neuron diseases" by Dr. U.B. Pandey, Pittsburgh, in which he discussed the role of protein FUS linked to both familial and sporadic forms of ALS.

We also attended the lecture of Dr. P. Srivastava from IIIT, Hyderabad, who presented the difference in male and female in making sense of desktop 360-degree virtual space. They found variable response in different sexes and related this with hormonal levels of testosterone. They also examined the interaction between gender type and interaction type.

Dr. T.C. Nag, AIIMS, New Delhi, presented the effect of iron overload and its consequence with age in retinal structural alteration and expression of iron regulatory proteins. It was found that iron accumulated with age, resulting in phagosome accumulation in RPE, damaged photoreceptors, thinning of outer segment and increased TUNNEL positive cells. In the next lecture, Dr. T. Srivastava from the University of Delhi explained the epigenetic 
regulation of glioma stem cell biology in the hypoxic microenvironment. The cancer stem cells, which are in few proportions in cancer cells, are found to be in hypoxic condition that causes cells to be in a demethylated state, leading to an exacerbated expression of oct-4 and Nanog. Dr. R. Pal from TIFR, Bengaluru, described the role of human IPSC for disease modelling and treatment of age-related macular degeneration. He used IPSC cell culture and differentiated RPE cells with prominent morphology that is, cilia processes and melanosomes. Functional analysis was done by calcium response and electrophysiological responses. The poster session was held from 4:40 to 5:30 PM near 7 pillars of wisdom. Sushant Kaushal received appreciation from scientists for his work on validation of thesis data. Abdul Ghani and Gurkeerat Kaur presented a talk on Yoga and its benefits in different diseases. They were also applauded by scientists and students.

October 31st, which was the last day of conference, started with a talk by Dr. Anita Jagota on "biological clock dysfunction in aging and Parkinson's disease". In her talk, she discussed her studies on purturbances in the functional integrity of circadian system with the neural degenerative changes in aging and Rotenone-induced Parkinson's disease rat model. Stoichiometric alterations obtained in the levels in the genes such as bmal1, per2, cry 1 and cry 2 were observed. Proteome analysis using MALDI
$\mathrm{TOF} / \mathrm{TOF}$ in relation to locomotor activity profiles has led to the identification of candidate biomarkers of aging and neurodegeneration, which will help in targeting novel treatments of circadian dysfunction for age-induced disorders and PD. The lecture of Dr. T.S. Roy, AIIMS, New Delhi on the topic "Age-related changes in the expression of glutamic acid decarboxylase (GAD67) and NMDA receptor (NMDA-R1) in the human inferior colliculus and another scientist Dr. K.K. Barhwal, AIIMS, Bhubaneswar, on the topic entitled salidroside as a potential pharmacological molecule for treatment of hypoxia induced neurodegeneration was delivered where he discussed the therapeutic effect of salidroside on various neurodegenerative diseases.

Overall, it was a unique experience. The conference enhanced our knowledge in Neuroscience, which included latest researches and advancement in its techniques. We also got an opportunity to meet different scientists from all over the world and were able to understand their research in this field.

\section{Disclosure Statement}

The authors declare that they have no conflicts of interest to disclose. 